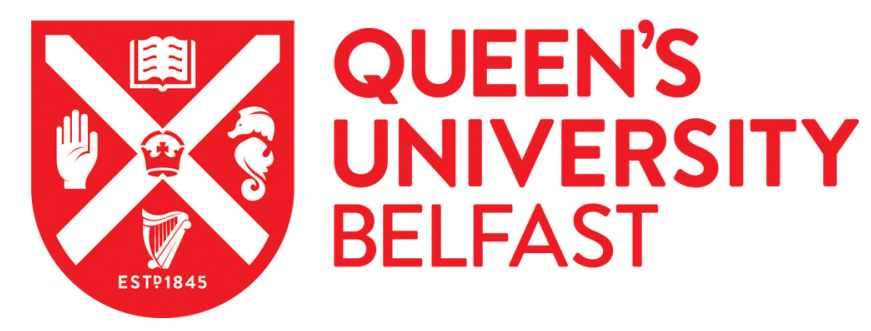

\title{
Nanoscale Dynamics of Superdomain Boundaries in Single-Crystal BaTiO3 Lamellae
}

Sharma, P., McQuaid, R., McGilly, L. J., Gregg, J. M., \& Gruverman, A. (2013). Nanoscale Dynamics of Superdomain Boundaries in Single-Crystal BaTiO3 Lamellae. Advanced Materials, 25(9), 1323-1330. https://doi.org/10.1002/adma.201203226

Published in:

Advanced Materials

Document Version:

Peer reviewed version

Queen's University Belfast - Research Portal:

Link to publication record in Queen's University Belfast Research Portal

Publisher rights

(c) 2013 WILEY-VCH Verlag GmbH \& Co. KGaA, Weinheim

This is the accepted version of the following article:Sharma, P, McQuaid, RGP, McGilly, LJ, Gregg, JM \& Gruverman, A 2013, 'Nanoscale Dynamics of Superdomain Boundaries in Single-Crystal BaTiO3 Lamellae' Advanced Materials, vol 25, no. 9, pp. 1323-1330, which has been published in final form at http://onlinelibrary.wiley.com/wol1/doi/10.1002/adma.201203226/abstract

\section{General rights}

Copyright for the publications made accessible via the Queen's University Belfast Research Portal is retained by the author(s) and / or other copyright owners and it is a condition of accessing these publications that users recognise and abide by the legal requirements associated with these rights.

Take down policy

The Research Portal is Queen's institutional repository that provides access to Queen's research output. Every effort has been made to ensure that content in the Research Portal does not infringe any person's rights, or applicable UK laws. If you discover content in the Research Portal that you believe breaches copyright or violates any law, please contact openaccess@qub.ac.uk. 
DOI: 10.1002/adma.201203226

\section{Nanoscale Dynamics of Superdomain Boundaries in Single-Crystal $\mathrm{BaTiO}_{3}$ Lamellae}

By Pankaj Sharma, ${ }^{{ }^{*}}$ Raymond G. P. McQuaid, ${ }^{2 *}$ Leo J. McGilly, ${ }^{2+}$ J. Marty Gregg, ${ }^{2 \dagger}$ and Alexei Gruverman ${ }^{1+}$

${ }^{1}$ Department of Physics and Astronomy and Nebraska Center for Materials and Nanoscience, University of Nebraska-Lincoln, Nebraska 68588-0299, U.S.A.

${ }^{2}$ School of Mathematics and Physics, Queen's University of Belfast, Belfast, BT7 1NN, United Kingdom

Keywords: $\mathrm{BaTiO}_{3}$ lamellae, domain boundaries, domain dynamics, piezoresponse force microscopy

A defining characteristic of ferroelectric materials is that the direction of the spontaneous polarisation can be reversed by application of a suitably oriented electric field. It is this bipolar property that suggests great potential for this material system in electronic device applications such as ferroelectric random access memory (FRAM) or ferroelectric field effect transistors (FeFET's). The mechanism for reorientation of polarisation involves the nucleation and growth of electric domains. However, although the key stages normally associated with domain-mediated polar switching have been understood for many decades, a definitive basic equation of motion for domain wall movement remains uncertain. The situation is further complicated by a growing realisation that switching need not only involve simple $180^{\circ}$ domain formation, but could also be facilitated by distinct bundles or groupings of domains, recently referred to as 'superdomains'. In this work, we observe the critical role of superdomains in switching thin single-crystal slices of $\mathrm{BaTiO}_{3}$ and derive effective

\footnotetext{
* These authors contributed equally to the paper

${ }^{\dagger}$ Current address: Ceramics Laboratory, EPFL-Swiss Federal Institute of Technology, Lausanne 1015, Switzerland

${ }^{\dagger}$ Electronic mail: m.gregg@qub.ac.uk

†Electronic mail: agruverman2@unl.edu
} 
equations of motion to describe the dynamics of superdomain boundaries under applied fields.

Pioneering domain imaging in bulk $\mathrm{BaTiO}_{3}$ crystals [1-3] in the 1950's revealed that $180^{\circ}$ polarization reversal in ferroelectric systems could be described by a three-stage switching process, analogous to that found in ferromagnets. The stages of switching were established as follows: firstly, formation of reverse polarized nuclei; secondly, rapid forward growth of the nuclei transforming into high aspect-ratio needle-like domains; and finally, slower sideways domain expansion leading to complete switching of the remaining crystal volume. The advent of experimental techniques which enable polarization mapping at the nanoscale, in particular piezoresponse force microscopy (PFM) [4] and transmission electron microscopy (TEM), have revealed that this behavior is by no means restricted to bulk crystals and can also be observed in nano-scale ferroelectrics at device-relevant dimensions, where polarization switching and retention is key to functionality [5-7].

However, such a simple picture of ferroelectric switching may alter whenever there is greater complexity in the domain microstructure. Recently, studies on microscale crystals of $\mathrm{BaTiO}_{3}[8]$ and $\mathrm{Pb}(\mathrm{Zr}, \mathrm{Ti}) \mathrm{O}_{3}(\mathrm{PZT})$ [9], that were machined from single-crystal boules using a focused ion beam (FIB) microscope, have revealed the critical role played by "superdomains", or bundles composed of $90^{\circ}$ stripe subdomains. Superdomains have been found to behave as distinct functional entities in their own right, facilitating flux-closure [10] and demonstrating the same Landau-Kittel scaling laws normally characteristic of individual conventional domains rather than domain packets [8]. This has been reiterated in other studies on PZT [11] and $\mathrm{BiFeO}_{3}[12]$ thin films, where ferroelectric switching has been seen to proceed by a cooperative reorganization of ferroelastic striped domain bundles. The existence of superdomain architectures has been central to the observation of several recent exotic phenomena including development of mesoscopic ferroelectric flux-closure domain 
patterns [10], enhanced conductivity at the core of flux-closure dipole arrangements [13], and room temperature switching of the net magnetization in a ferromagnetic/ferroelectric bilayer by reversing the net polarization of a ferroelectric superdomain [14]. To harness any potential additional functionalities offered by superdomain structures, it is critical to assess their fieldinduced switching dynamics, and in particular to find out how it compares with conventional $180^{\circ}$ ferroelectric switching. The most relevant detailed switching studies have thus far been conducted using local scanning probe techniques, primarily PFM, and typically involve highly inhomogeneous electric fields applied locally through the PFM tip [11]. Some insight into superdomain switching in more device-relevant capacitor-type geometries has been reported using a sequence of planar field switching pulses followed by PFM imaging $[15,16]$. Additionally, work by the groups of Taheri [17,18], Ikuhara [19,20] and Shang [21], have focused on using an in-situ TEM approach to image real-time domain wall motions in systems where complex domain structures and hierarchies exist. Whilst the remarkable spatial and temporal resolutions associated with TEM allowed domain wall motion to be probed from nanometer to micrometer length scales, an equation of motion describing individual boundary movement was not established.

Here we report in-situ, i.e. in the presence of an external switching d.c. bias applied through co-planar electrodes, PFM imaging of the nucleation and field-driven dynamics of superdomains consisting of bundles of ferroelastic $a$-c-domains. We make several key observations: firstly, it appears that the simple three-stage reversal process has been reproduced at the superdomain level i.e. via nucleation and growth of $a-c$ domain packets rather than single domains; secondly, that the superdomain boundary velocity is not constant for the duration of switching; and, thirdly, that the velocity is maximum at the moment the planar switching field is applied and rapidly decays monotonically thereafter while the field is still on. Treating the superdomain boundary motion in the framework of an elastic wall 
moving through a damping medium, this behavior can be described by a standard equation of motion. The boundary dynamics seem to be analogous to ballistic projectile motion where, after initial launch, the boundary slows down to rest due to the drag associated with the viscous medium. However, we propose that true ballistic motion of the superdomain boundary is unphysical, and instead suggest that the initial driving force from the applied electric field is rapidly opposed by a counter-balancing force from an uncompensated depolarizing field of newly switched domains, that quickly nullifies the applied driving field for the remaining duration of its application.

Thin $\{100\}_{\text {pseudocubic(pc) }}$-oriented single-crystal $\mathrm{BaTiO}_{3}$ slices or lamellae, nominally $300 \mathrm{~nm}$ in thickness, were cut using an established FIB milling methodology [22-24]. They were then transferred onto pre-patterned platinum thin film co-planar electrode structures (on single crystal $\mathrm{MgO}$ substrates) and oriented such that $\langle 100\rangle_{\mathrm{pc}}$ directions were approximately parallel and perpendicular to the edges of the patterned interelectrode gaps. Samples were thermally annealed in air (at $700^{\circ} \mathrm{C}$ ) and then etched in $\mathrm{HCl}$ of $3 \mathrm{M}$ concentration. This processing was found to fully recover ion-beam induced surface damage and produce a surface quality suitable for PFM investigation. Finally, lamellae were secured in place using local electron-beam deposition of platinum electrodes in a dual-beam FIB system [10]. PFM measurements were performed using a commercial atomic force microscope Asylum Research MFP-3D system by applying a high frequency $(670 \mathrm{kHz}$ for lateral PFM and 330 $\mathrm{kHz}$ for vertical PFM) modulating voltage $\left(1.5 \mathrm{~V}_{\mathrm{pp}}\right)$ to conductive PtIr-coated silicon cantilevers.

The experimental setup is schematically illustrated in Figure 1a and the PFM images of the superdomain boundary separating bundles of $a$-c-domains are shown in Figures $1 \mathrm{~b}$ to 1d. In the lateral PFM (LPFM) images in Figure $1 b$ and 1c, it can be seen that the in-plane polarization ( $a$-subdomains), oriented perpendicular to the electrodes, reverses direction 
across the boundary. The vertical PFM (VPFM) amplitude image in Figure 1d reveals a complex $a$-c-domain arrangement that persists on both sides of the superdomain boundary. Unambiguous mapping of the precise topology of the superdomain boundary using VPFM phase information was complicated by low signal-to-noise issues. PFM images captured during switching, discussed later, strongly imply a continuous boundary suggesting that the $c$ subdomains also switch across this boundary. Additionally, in some rare cases, lamellae prepared using the same methodology can exhibit an $a$-a-subdomain structure; here a continuous superdomain boundary can be unambiguously mapped by LPFM imaging (Figure S1a,b, Supporting Information). We believe this provides compelling evidence for a continuous superdomain boundary in the $a$-c-subdomain case and is discussed further in the Supporting Information.

To investigate the formation and dynamics of the superdomain boundaries under the applied electric field, initially a saturating in-plane field was applied across the lamella. As a result, all superdomain boundaries were flushed out leaving a single $a$-c-domain bundle (elucidated by VPFM in Figure 2a) where in-plane $a$-subdomains were aligned parallel to the field. We found that after the external field was removed, a needle-like $a$-c-domain bundle (superdomain) with the reverse $a$ - (and $c$-) subdomain orientation spontaneously nucleated at the electrode. It subsequently propagated forward until it reached the opposite electrode and then continued to expand sideways forming two planar superdomain boundaries (Figure 2b). Schematic illustrations of the switching process are shown in Figures $2 \mathrm{~d}$ and 2e. We again stress the persistence of the $a$ - $c$-domain stripes throughout the backswitching process (see Figure $2 \mathrm{a}$ and $2 \mathrm{c}$ ). The observed switching process is completely analogous to the switching behavior observed by Merz except that it occurs at a superdomain level, rather than at an individual domain level. This is quite distinct from the picture of planar switching of stripe domain packets in, for example, $\mathrm{BiFeO}_{3}$ films by Heron et al [14] where phase field 
modeling portrays a two-step switching process initiated by independent nucleation events within alternate domain stripes, rather than the propagation of a continuous switching wavefront.

Once created, the sideways motion of these complex superdomain boundaries can be induced by application of a planar switching field and observation of their switching dynamics in real-time via in-situ PFM mapping can be done. Figure 3a reveals the fieldinduced displacement of the superdomain boundary as a function of time. Here, each scan line represents a time lapse of $2.5 \mathrm{~s}$ (at the scan rate of $0.4 \mathrm{~Hz}$ ). It is important to emphasize that the apparent distortion in Figure 3a is not an actual deformation of the boundary but is simply a representation of its time-dependent position in the applied field resulting from lineby-line tip rastering during the PFM image acquisition. We envisage the true boundary motion to be as depicted schematically in Figure $3 \mathrm{~b}$. Given that the boundary remains perpendicular to the electrode edges, we have been able to monitor its dynamics by representing the net boundary position as a point of intersection between the boundary and a single scan line. It is worth highlighting that two superdomain boundaries can come together and annihilate (Figure 3a panels (iii)-(vi)), in complete analogy to the disappearance of $180^{\circ}$ walls in the Merzian type switching. The superdomain boundaries are observed to slow down as they come into close proximity to each other, suggesting repulsive interaction between domains of the same polarity.

Using this approach, we monitored the time-dependent position of the superdomain boundary at different external voltages (Figure 4a). An oscillation-like pattern superimposed on the boundary motion profile in the PFM images is due to the morphology of the $a-c$ stripe subdomains that form the superdomain boundary. By tracking the domain boundary position over several PFM capture frames we were able to extend the period of observation to several minutes. Results of the PFM data analysis from Figure 4a are shown in Figure 4b where the 
boundary position is plotted as a function of time and voltage. Figure $4 \mathrm{c}$ shows the scheme for extracting the boundary position as a function of time (presented in Figure $4 \mathrm{~b}$ ) based on knowledge of the spatial position and collection time for each pixel. Lower-bound estimates for the initial velocity of the boundary as a function of voltage are presented in Figure S2 (Supporting Information) using time gradients from Figure 4b.

A common feature of the superdomain boundary dynamics is a large jump from its initial position at the moment the external field is applied followed by a much slower progression thereafter. This distinct relatively high speed motion lasted a period of a few seconds at most (see Figure $4 \mathrm{~d}$ for a typical velocity/time profile). The magnitude of this initial jump was found to increase with magnitude of the applied voltage (field). Two surprising observations can be made: firstly, that the velocity of the boundary does not remain constant for the entire duration over which the switching field is applied; secondly, that the velocity is maximum at the moment the field is initially applied $\left(v_{0}\right)$ (Figure $\left.4 \mathrm{~d}\right)$ and rapidly decreases thereafter either to zero or some steady-state velocity that is negligible in comparison to $v_{0}$. This behavior closely resembles the dynamics of planar $180^{\circ}$ domain walls observed in uniaxial ferroelectric crystals of lead germanate and gadolinium molybdate [25].

Fitting the time dependence of polarization reversal resulting from the motion of a single superdomain boundary using statistical (Kolmorogov-Avrami-Ishibashi [26,27] or nucleation-limited switching $[28,29])$ models seems inappropriate, since their applicability is associated with a large number of randomly distributed nucleation events. Furthermore, when we attempt to fit our data using the KAI approach we find satisfactory fits only when an unphysical dimensionality of domain growth is assumed i.e. much less than unity (see Supporting Information and Figure S3). Since we are dealing with the sidewards motion of a pre-existing planar boundary, we instead follow Kittel's proposal that the equation of motion 
(EOM) for domain wall propagation used in magnetics may also be applicable for $\mathrm{BaTiO}_{3}$ [30], taking the general form:

$$
m \frac{d^{2} x}{d t^{2}}+\beta\left(\frac{d x}{d t}\right)^{n}+k x=F
$$

where $m$ is an effective boundary mass, $\beta$ is a viscous damping parameter, $n$ is an exponent that determines whether damping scales linearly or quadratically with velocity, $k$ is a restoring force constant, and $F$ is the effective force exerted on the boundary by the driving field.

There are several specific forms of Equation (1) that offer reasonable fits to our observed data. Perhaps the simplest is captured when damping is assumed to vary quadratically with velocity $(n=2)$. Under these circumstances, best fits give a driving force of zero within uncertainty (see Figure S4, Supporting Information), and have no need for a restoring force term. Thus the EOM is simplified to:

$$
\frac{d^{2} x}{d t^{2}}=-b\left(\frac{d x}{d t}\right)^{2}
$$

This expression is characteristic of ballistic projectile motion (e.g. the horizontal trajectory of a fired bullet) where $b$ is a reduced damping parameter. We parenthetically note that inferior fits were obtained when a purely ballistic scenario with linear, rather than quadratic, damping (i.e. $\mathrm{n}=1$ ) was assumed (see Supporting Information and Figure S5 for details). The solution to Equation (2) for boundary position $(x)$ is logarithmic in time [31], having the form:

$$
x(t)=\frac{1}{b} \log _{e}\left(1+v_{0} b t\right)
$$


where $v_{0}$ is the initial launch velocity (at time $t=0$ ) of the boundary. Best fits for the measured position vs. time data and the extracted values for $b$ as a function of voltage are plotted in Figure 5a,b. Rather non-intuitively, this description depicts a boundary, which is launched with a finite initial velocity at the moment the field is applied, but experiences no effective driving force thereafter. We stress that the EOM is only capable of describing the motion after this initial 'launch' and the associated $v_{0}$ must be entered into the description as an initial condition. This limitation is exemplified whenever we try to incorporate a driving force into our EOM description. We find that our data can also be well represented by a driven EOM (i.e. $F>0$ in Equation (1)) in the case where linear velocity damping is assumed:

$$
m \frac{d^{2} x}{d t^{2}}+\beta \frac{d x}{d t}+k x=F
$$

having solutions of the form:

$$
x(t)=\frac{f t}{b}+\left(\frac{v_{0}}{b}-\frac{f}{b^{2}}\right)\left(1-e^{-b t}\right)
$$

where $f$ is here a reduced force. Critically however, this does not replace the need for a large preassumed initial velocity to be entered as an initial condition to the EOM. The driving force term is only really pertinent for determining the modelled terminal velocity $\left(v_{t e r}\right)$ of the boundary, analytically determined as the ratio $f / b$. We emphasize that this is only really relevant for modelling the slow-movement portion of the boundary motion and does not explain the rapid early-time development. The modelled profiles for $f$ and $b$ as a function of voltage, extracted from Figure 5c, are shown in Figure 5d. The value of $b$ is almost a constant (within uncertainty) in this scenario, suggesting the damping response of the medium (as experienced by the moving boundary) to be the same across the studied voltage range. That $f$ 
shows a nearly linear development with voltage seems reasonable when we consider that in an ideal capacitor geometry $F \propto E=V / d$ (with $d$ as plate separation).

The type of logarithmic position-time dependence for domain wall dynamics described by Equation (3) has been inferred previously in PZT capacitors through impedance spectroscopy data [32] and through direct imaging of domain wall motion [33]. In both cases, ballistic motion was invoked. However, in the present experiments we notice that superdomain boundary motion ceases whenever the applied field is removed (in fact often some degree of backswitching occurs as can be seen in Figure 4a), indicating that forward motion is only sustained for the duration over which the field is applied. A similar conclusion could be drawn from Kim et al's experiments [33], where the time over which the field is applied determines the wall position, not the total time between the initial application of the field and the imaging of the domain wall. Thus, although the superdomain boundary dynamics may exhibit some characteristics of the ballistic behavior, this mechanism is inconsistent with the fact that the domain wall stops after the external voltage is turned off.

We, therefore, consider the possibility that the net driving force exerted on the domain boundary may not be constant even in the presence of a nominally homogenous switching field. We believe that that the net force acting on the boundary varying as a function of space and/or time is more likely to account for its apparent deceleration rather than through an intrinsic inertial response to a constant driving force (as suggested by an EOM that is $2^{\text {nd }}$ order in time). Catalan et al [34] argue however that since ferroelectric domain walls move by virtue of ionic shifts they should indeed exhibit an inertial response. By contrast, ferromagnetic domain wall motion, facilitated by spin-flipping, involves movement of zero mass and therefore no inertial response is anticipated. Somewhat paradoxically, the majority of experimental evidence points to the converse situation being true, where wall resonance 
$[35,36]$ and ballistic overshoot phenomena $[37,38]$ are attributed to an inertial-type response of domain walls in magnetic systems and can be characterized by an associated effective mass. For ferroelectrics however, the present study suggests that whist it may be tempting to extrapolate the observed signature of ballistic dynamics to inertial behavior, this may not be appropriate.

In the present experiment, to explain the observed dynamics, we can envisage the following scenario. As soon as the planar field is applied, the boundary rapidly attains its initial velocity on timescales shorter than the PFM time resolution (this seems reasonable given that domain response to applied fields has been regularly observed on nanosecond timescales [39-41]). Subsequently, an opposing force is generated in the system that counteracts the driving force from the applied field. This opposing force should arise with a characteristic time that is larger than the response time of the domain boundary to the applied field (hence allowing for the rapid initial displacement). Thus a progressive decrease in the net forward driving force on the domain wall would result in boundary movement with progressively diminishing velocity and could explain the apparent ballistic dynamics even in the presence of a constant applied driving force.

In bulk electroded $\mathrm{BaTiO}_{3}$ crystals, the development of charged surface layers that inhibit domain wall motion has been proposed to account for velocity transients that appear during pulsed switching experiments $[42,43]$. In our case, we suggest that such an opposing force is likely to be related to the uncompensated depolarizing field of the newly switched $c$ subdomains involved in the superdomain boundary dynamics [25]. Displacement of the superdomain boundary from its initial position under the applied planar electric field is accompanied not only by the in-plane reversal of $a$-domains but also, as a consequence of the charge neutrality of the $a$ - $c$ domain boundary, by the out-of-plane reversal of $c$-subdomains in the volume behind the moving boundary (Figure 6a). Note that, in the absence of electrodes, 
the predominant mechanism of polarization screening of $c$-domains is external, i.e. via adsorption of dipoles and charged species from environment (Figure 6b). Calculation of the out-of-plane electric field at the free surface of the lamella assuming complete external polarization screening shows that the field distribution is nonuniform [43]. Indeed, a superposition of the electric fields of bound polarization and screening charges results in an electric field distribution with a maximum in the close proximity to the $c$-domain boundary and almost zero away from it (Figure 6c). Initial quick acceleration of the superdomain boundary is facilitated by this local maximum of the electric field. However, since the external screening of the out-of-plane polarization is a fairly slow process (with typical characteristic time of the order of minutes), a quick initial displacement of the boundary will result in the build-up of the uncompensated depolarizing field of $c$-domains behind the moving superdomain boundary thereby slowing down their switching. Since the superdomain is composed of coupled $a$-c-domains, the increasing depolarizing field will also slow down the overall boundary movement. In Figure 4a, it can also be seen that planar field application seems to increase the amplitude of the boundary's corrugated profile. We speculate this 'roughening' of the boundary could be due to $a$-subdomain boundaries advancing under field application whilst the $c$-subdomain boundaries resist movement. Thus, the domain dynamics is determined by the relationship between the rate of screening charge accumulation and superdomain boundary velocity under the applied electric field. The same uncompensated depolarizing field is responsible for the relaxation of the superdomain boundary toward its initial position after the external field is turned off (Figure 4a).

To summarise, we have used PFM to perform in situ investigation of the superdomain boundaries dynamics under homogeneous external fields in thin single-crystalline lamellae of $\mathrm{BaTiO}_{3}$. The nucleation and expansion of superdomains - distinct bundles of $a-c$ stripe domains - has been directly observed. The motion of superdomain boundaries was found to 
be closely analogous to ballistic projectile dynamics. However, pure ballistic domain wall motion was ruled out on the grounds of being unphysical. Instead, we have proposed a time variation of the effective driving force for domain wall motion which results from interplay between applied and unscreened depolarizing fields.

\section{Acknowledgements}

The authors acknowledge financial support from the Engineering and Physical Sciences Research Council (EPSRC) and the National Science Foundation (NSF) under the Materials World Network (MWN) program (NSF Grant No. DMR-1007943 and EPSRC Grant No. EP/H047093/1). R.G.P.McQ., J.M.G. and A.G thank the Leverhulme Trust for international network funding (F/00 203/V). R.G.P.McQ., L.J.McG. and J.M.G. acknowledge support from the Department of Employment and Learning (DEL). R.G.P.McQ. acknowledges support from the Dunville Studentship scheme. P.S. and A.G. acknowledge support from the NSF Materials Research Science and Engineering Center (Grant No. MRSEC DMR-0820521). The authors are grateful to Prof. J. F. Scott for valuable discussions. 


\section{Figure Captions}

Fig.1. (a) Schematic illustration of the co-planar switching geometry used to apply planar d.c. switching fields. A probing tip scanning between the electrodes images domains in PFM mode. (b) Lateral PFM amplitude and (c) phase of a superdomain boundary separating two $a$ $c$-domain bundles. (d) Vertical PFM amplitude image of the same boundary showing explicitly the ferroelastic domain stripes on either side of the boundary elucidated in (b) and (c). In (b)-(d) arrow labels on cantilever motifs indicate the direction of sensed polarization in the respective imaging modes.

Fig.2. (a) VPFM amplitude image of the initial domain structure of the $\mathrm{BaTiO}_{3}$ lamella indicating the presence of a single superdomain. (b) LPFM amplitude (left panels) and phase (right panels) imaging of the area marked by a dotted line in (a) showing nucleation and growth of a new superdomain. Black arrows to the right of amplitude panels indicate the slow scan direction during PFM imaging (which could be used to deduce the time flow direction). In (i) and (ii) a small nucleated superdomain is revealed by the local reduction in LPFM amplitude signal but is not yet resolved in the phase signal. In (iii) the growth of a needle superdomain is apparent and the lateral movement of two distinct boundaries is observed in (iv) and (v). (c) VPFM amplitude image of the domain structure after backswitching where one of the superdomain boundaries is faintly visible. (d),(e) A schematic illustration of the domain switching process visualized in (b).

Fig.3. (a) In-situ LPFM amplitude (left panels) and phase (right panels) imaging showing time-dependent displacement of the superdomain boundary under the planar external field. Black arrows to the right of amplitude panels indicate the slow scan direction during PFM imaging. The initial stationary position of a superdomain boundary in the absence of applied field is mapped in (i) and the moment the field is applied is marked in panel (ii) by the white 
arrow. A second boundary is seen to move into the field of view in panels (iii)-(v). In panel (vi) no boundaries are visible indicating the two superdomain boundaries have mutually annihilated. (b) Schematic representation of the boundary movement depicting a rectilinear displacement of the superdomain boundary under applied planar field (movement direction denoted by yellow-green buried arrow. (ii). Labelled arrows $\underline{\mathrm{E}}$ and $\underline{\mathrm{P}} / /$ indicate directions of applied field and superdomain in-plane polarization component, respectively.

Fig.4. (a) A sequence of in-situ LPFM images showing the superdomain boundary dynamics under the influence of a planar switching field as a function of an increasing pulse magnitude $(0.6 \mathrm{~V}, 1.0 \mathrm{~V}$ and $1.2 \mathrm{~V})$. Red arrows designate the moments when the field is applied and blue arrows when it is turned off. (b) Position of the superdomain boundary as a function of time for several voltages. Inset shows boundary position plotted on a logarithmic time axis. (c) A sketch illustrating the approach used to reconstruct the position of the domain boundary as a function of time. Each image pixel with $x, y$ coordinates and polarization $\underline{\mathrm{P}}$ in the PFM map has a unique capture time, t. (d) Typical form of the velocity/time profile obtained by differentiating position/time data such as in (b).

Fig.5 (a) Model fits obtained assuming a ballistic EOM as described by Equation (3) overlaid on measured boundary position vs. time data as a function of voltage. (b) Modelled reduced damping parameter, $b$, extracted from fits in (a), as a function of voltage. (c) Model fits obtained assuming a driven EOM with linear velocity damping as described by Equation (5). (d) Reduced damping and driving force parameters ( $b$ and $f$ respectively), as extracted from fits in (c), plotted as a function of voltage.

\section{ALEXEI CAN YOU SHORTEN FIGURE 6 CAPTION?}


Fig.6. (a) Schematic representation of a superdomain boundary and its displacement (illustrated by the dotted line) under the applied electric field. For clarity, only out-of-plane polarization components $\left(\underline{\mathrm{P}}_{\perp}\right)$ on either side of the boundary are indicated. (b) A sketch illustrating charge distributions for the static and displaced domain boundaries. In equilibrium, bound charges associated with the out-of-plane polarization are compensated through adsorption of surface charges. Instantaneous displacement of the boundary leads to a non-equilibrium electrostatic situation where the distribution of the adsorbed charges immediately behind the shifted domain wall (dotted boundary) is not changed from the equilibrium state due to a slow relaxation time and is the same as that of the bound charge. (c) A calculated distribution of the normal component of the electric field resulting from the superposition of the fields of bound polarization and screening charges in the vicinity of the domain boundary. 


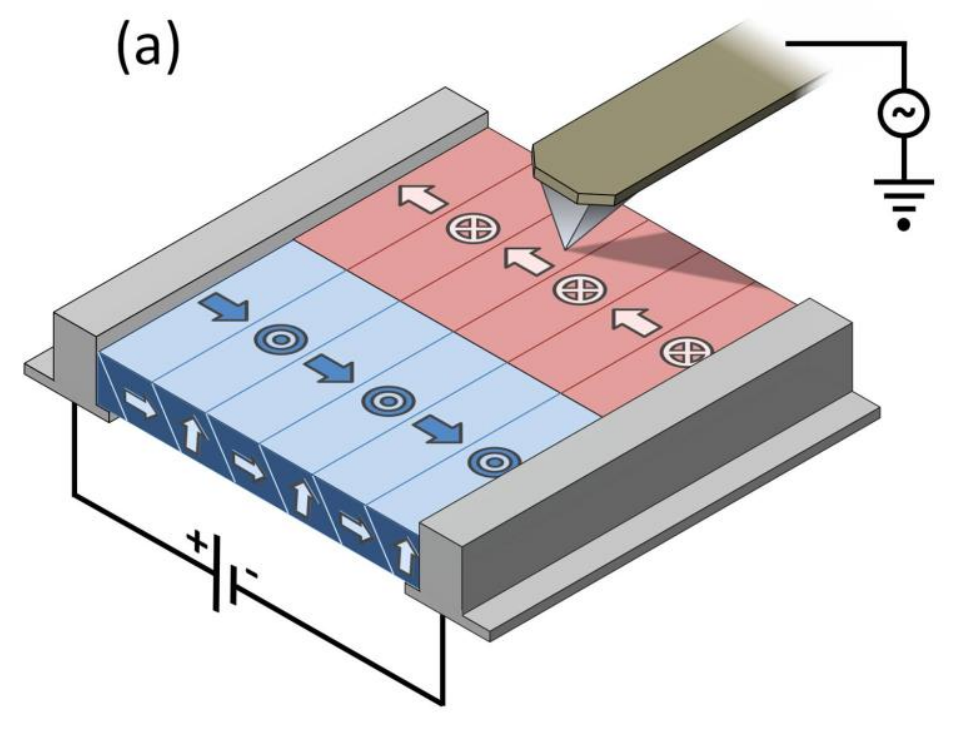

(b)

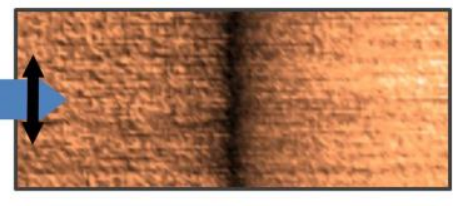

(c)

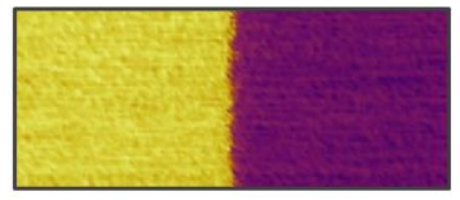

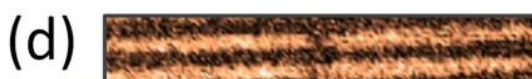

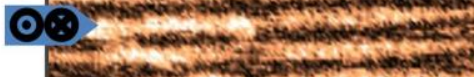

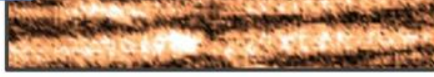
$\overline{0.5 \mu \mathrm{m}}$

Fig. 1 
(a)

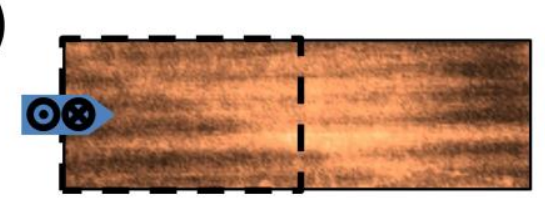

(b)

(i)

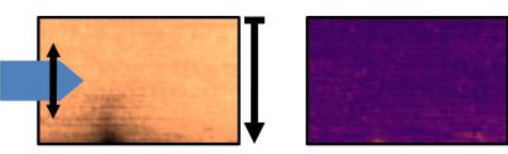

(ii)

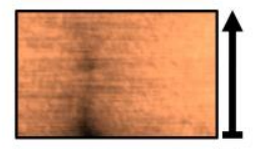

(iii)

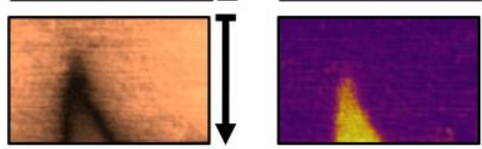

(iv)

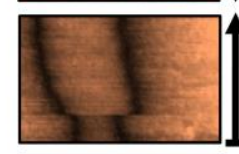

(v)

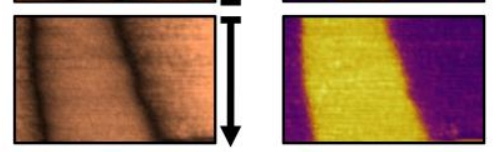

(c)

$$
1 \mu \mathrm{m}
$$

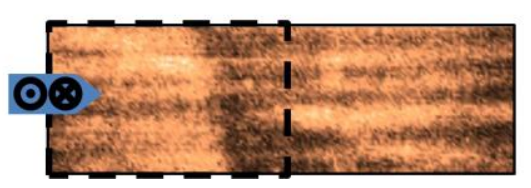

(d)
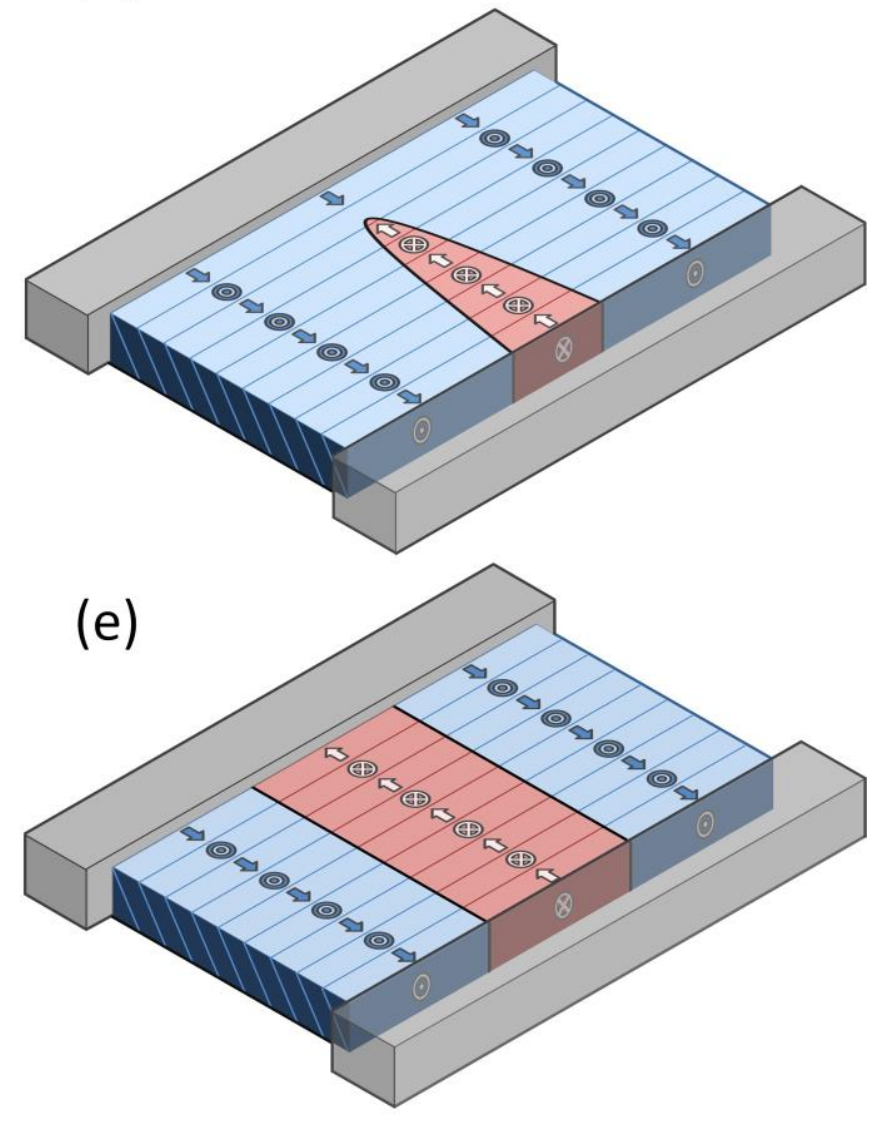

Fig. 2 

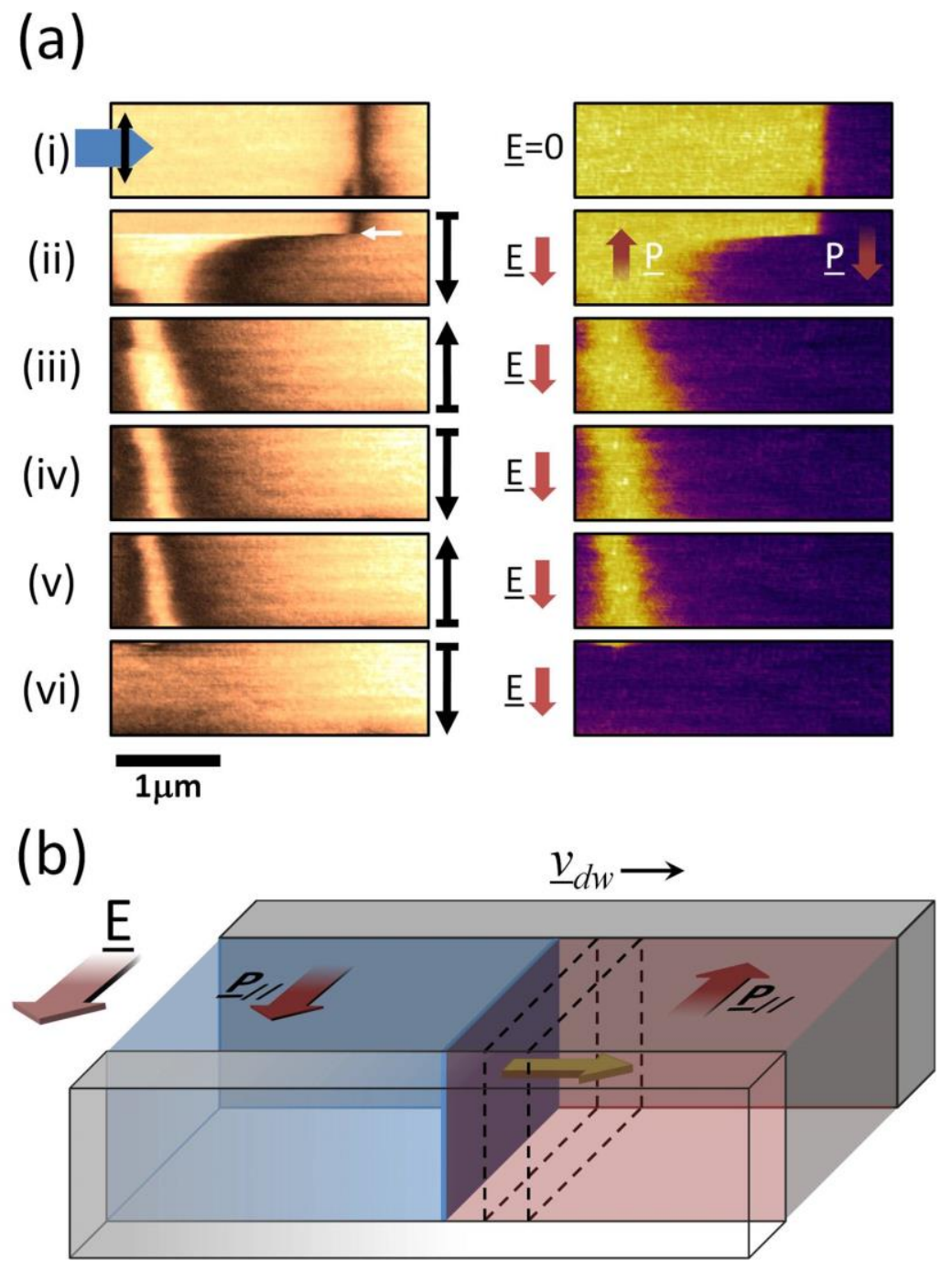

Fig. 3 
(a)

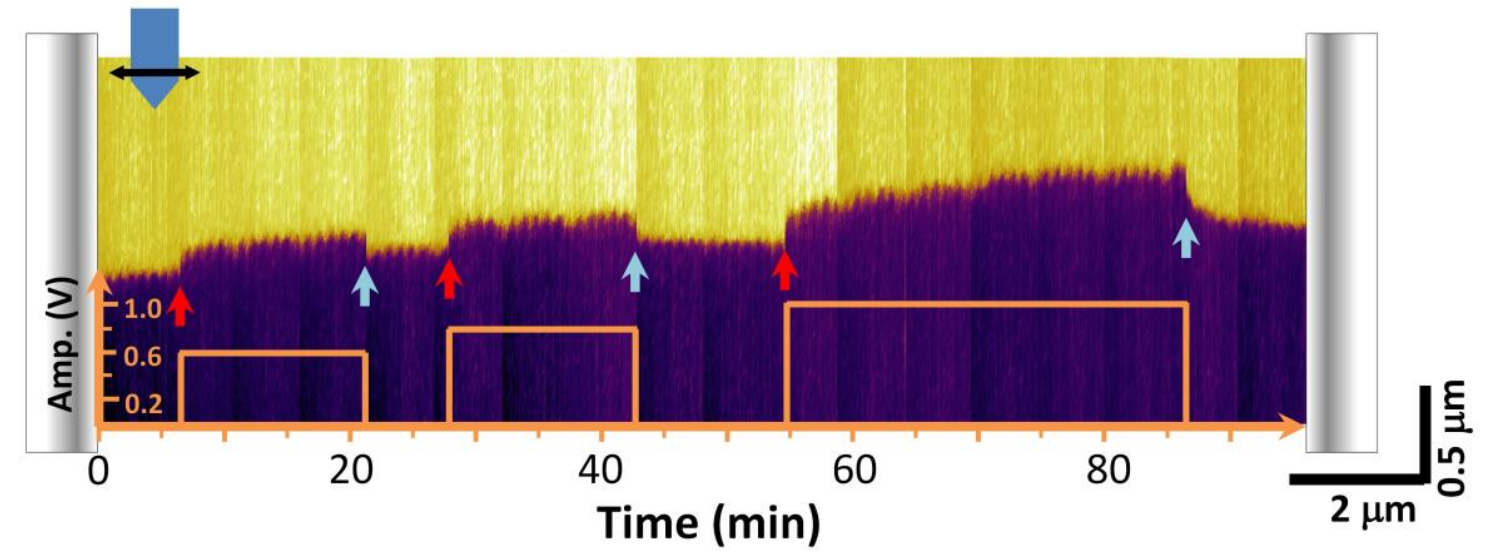

(b)

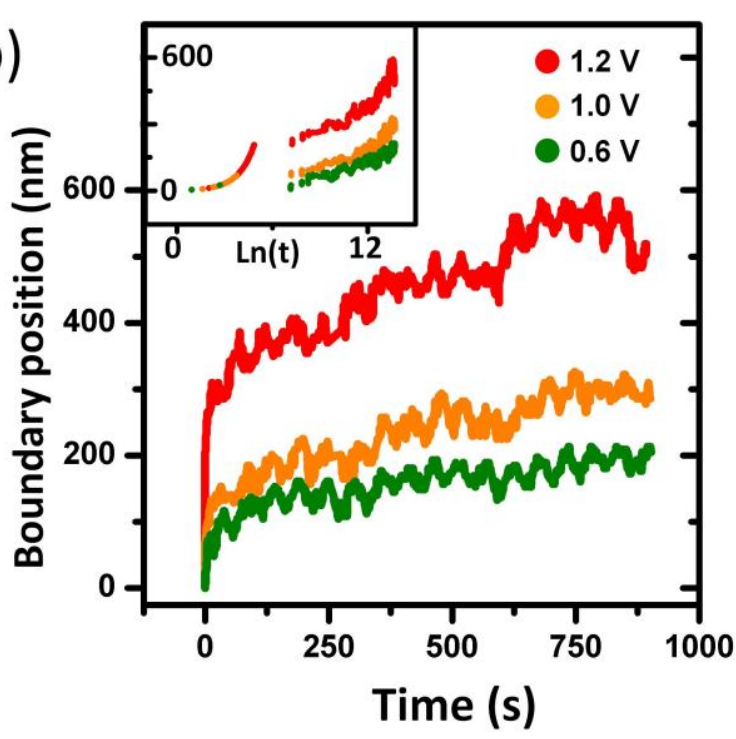

Fig. 4

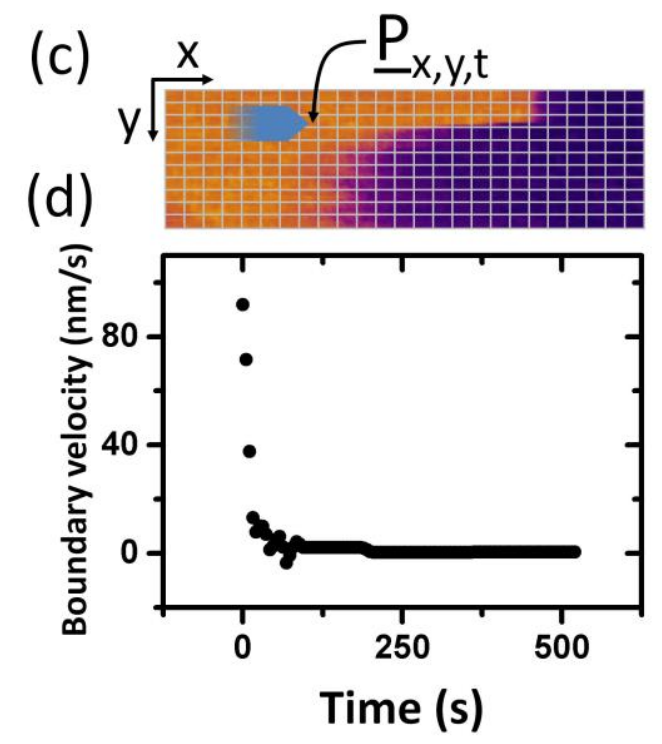


(a)

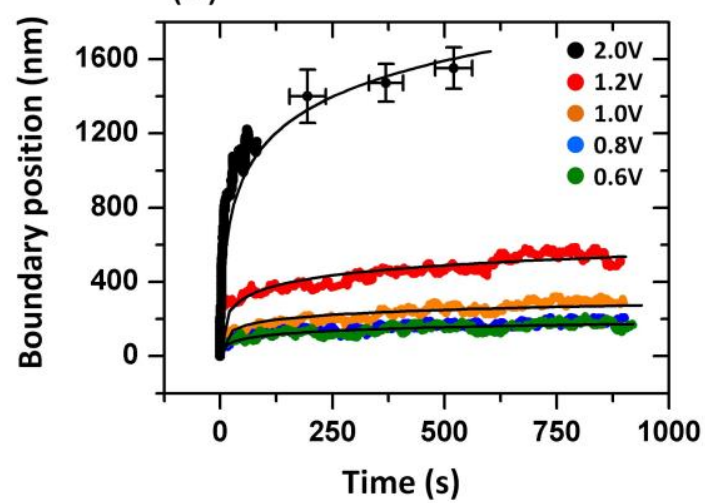

(c)

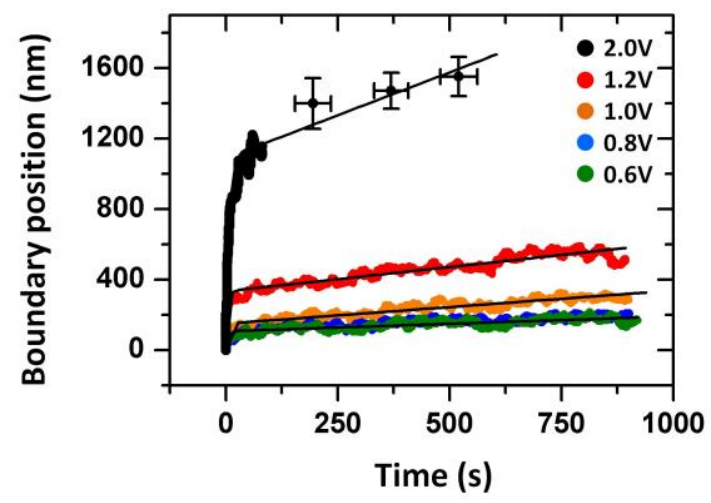

(b)
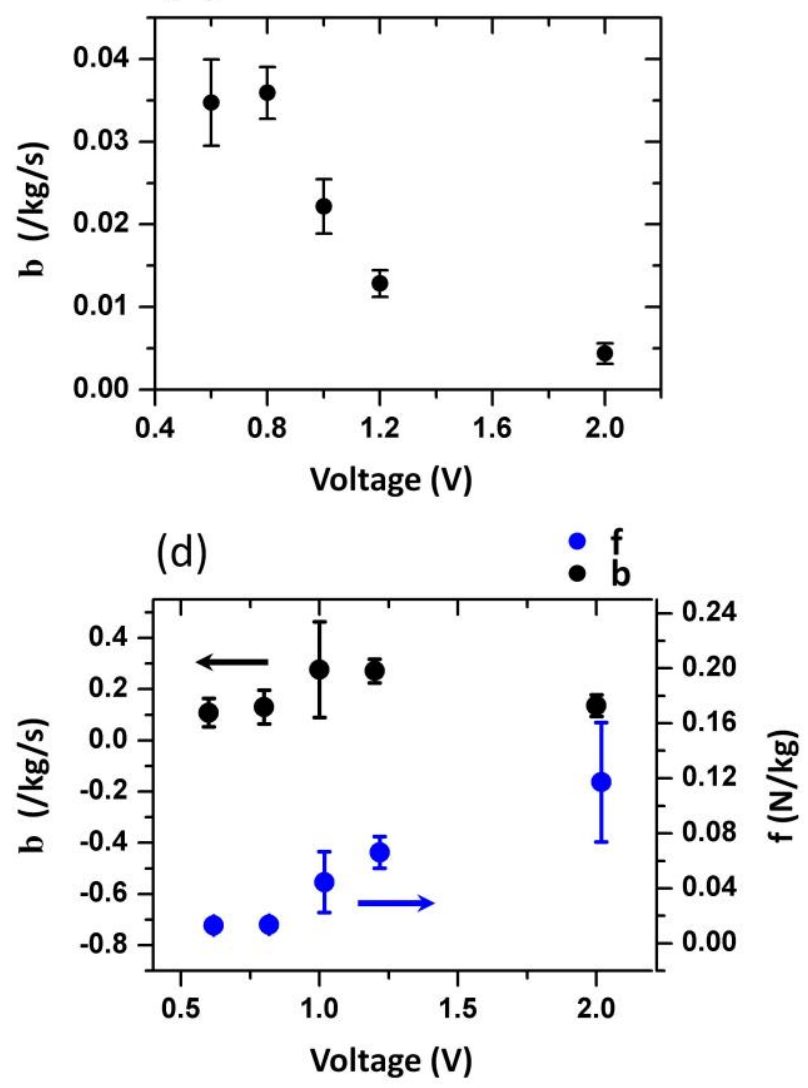

Fig. 5 
(a)
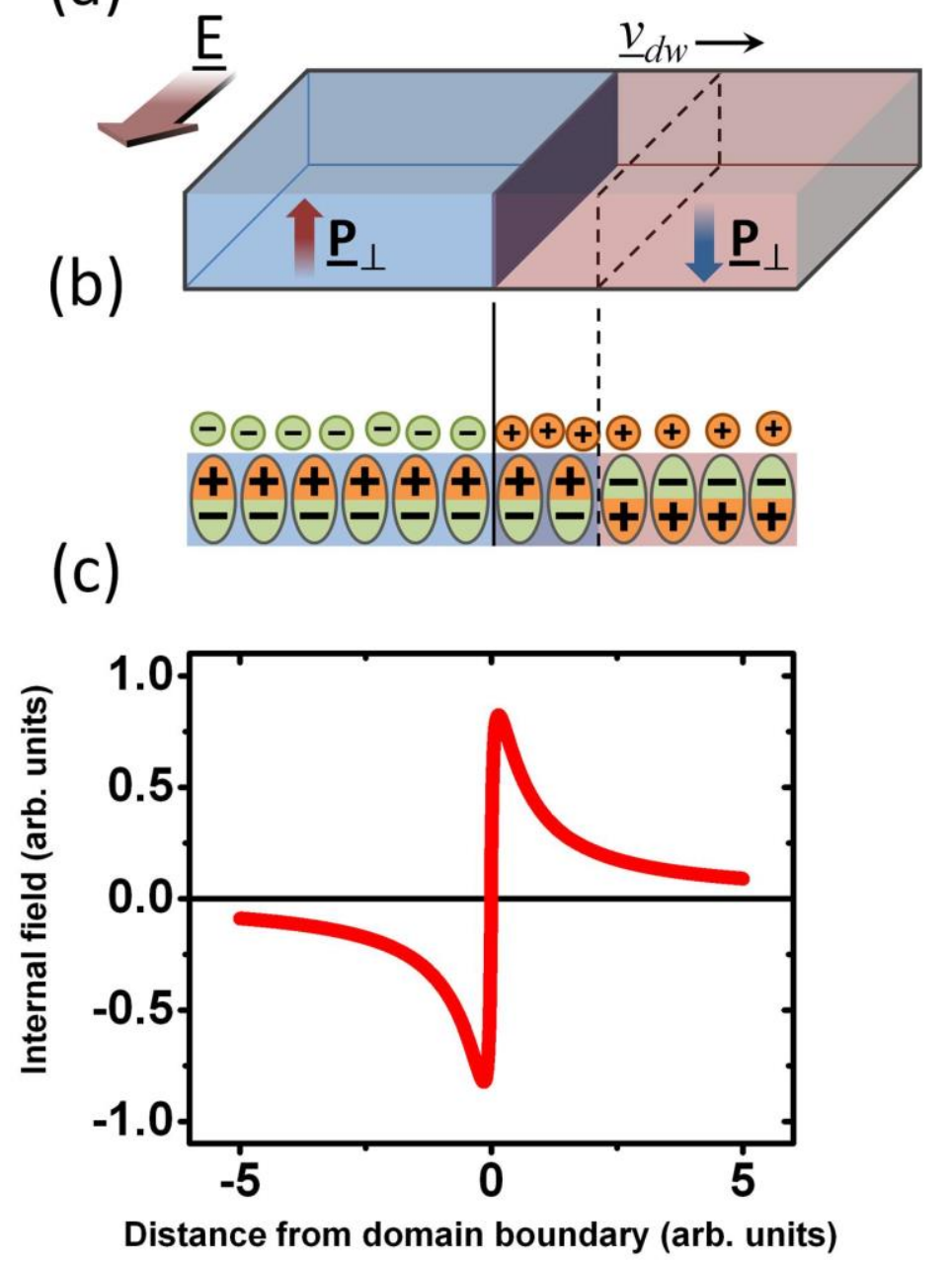

Fig. 6 


\section{References}

1 W. J. Merz, Phys Rev. 1954, 95, 690.

2 R. C. Miller, A. Savage, Phys. Rev. 1958, 112, 755.

3 R. C. Miller, Phys. Rev. 1958, 111, 736.

4 A. Gruverman, O.Auciello, H.Tokumoto, Annu. Rev. Mater. Sci 1998, 28, 101.

5 P. Gao, C. T. Nelson, J. R. Jokisaari, S.-H. Baek, C. W. Bark, Y. Zhang, E. Wang, D. G. Schlom, C.-B. Eom, X. Pan, Nat. Commun. 2011, 2, 591.

6 C. T. Nelson, P. Gao, J. R. Jokisaari, C. Heikes, C. Adamo, A. Melville, S.-H. Baek, C. M. Folkman, B. Winchester, Y. Gu, Y. Liu, K. Zhang, E. Wang, J. Li, L.-Q. Chen, C-B. Eom, D. G. Schlom, X. Pan, Science 2011, 334, 968.

7 N. Balke, M. Gajek, A. K. Tagantsev, L. W. Martin, Y.-H. Chu, R. Ramesh, S. V. Kalinin, Adv. Func. Mat. 2010, 20, 3466.

8 L. J. McGilly, J. M. Gregg, Appl. Phys. Lett. 2011, 98, 132902.

9 L. J. McGilly, J. M. Gregg, Nano Lett. 2011, 11, 4490.

10 R. G. P. McQuaid, L. J. McGilly, P. Sharma, A. Gruverman, J. M. Gregg, Nat. Commun. 2011, 2, 404.

11 Y. Ivry, N. Wang, D. Chu, C. Durkan, Phys. Rev. B 2010, 81, 174118.

12 N. Balke, S. Choudhury, S. Jesse, M. Huijben, Y. H. Chu, A. P. Baddorf, L. Q. Chen, R. Ramesh, S. V. Kalinin, Nat. Nanotechnol. 2009, 4, 868-875.

13 N. Balke, B. Winchester, W. Ren, Y. H. Chu, A. N. Morozovska, E. A. Eliseev, M. Huijben, R. K. Vasudevan, P. Maksymovych, J. Britson, S. Jesse, I. Kornev, R. Ramesh, L. Bellaiche, L.-Q. Chen, S. V. Kalinin, Nat. Phys. 2012, 8, 81.

14 J. T. Heron, M. Trassin, K. Ashraf, M. Gajek, Q. He, S. Y. Yang, D. E. Nikonov, Y.-H. Chu, S. Salahuddin, R. Ramesh, Phys. Rev. Lett. 2011, 107, 217202.

15 R. Guo, L. You, L. Chen, D. Wu, J. Wang, Appl. Phys. Lett. 2011, 99, 122902. 
16 L. You, E. Liang, R. Guo, D. Wu, K. Yao, L. Chen, J. Wang, Appl. Phys. Lett. 2010, 97, 062910.

17 C. R. Winkler, A. R. Damodaran, J. Karthik, L. W. Martin, M. L. Taheri, Micron, 2012, $43,1121$.

18 C. R. Winkler, M. L. Jablonski, A. R. Damodaran, K. Jambunathan, L. W. Martin, M. L. Taheri, J. Appl. Phys. 2012, 112, 052013.

19 Y. Sato, T. Hirayama, Y. Ikuhara, Phys. Rev. Lett. 2011, 107, 187601.

20 Y. Sato, T. Hirayama, Y. Ikuhara, Appl. Phys. Lett. 2012, 100, 172902.

21 X. Tan, H. Hea, J.-K. Shang, J. Mater. Sci. 2005, 20, 1641.

22 L.-W. Chang, M. McMillen, F. D. Morrison, J. F. Scott, J. M. Gregg, Appl. Phys. Lett. 2008, 93, 132904.

23 R. G. P. McQuaid, L.-W. Chang, J. M. Gregg, Nano Lett. 2010, 10, 3566.

24 A. Schilling, T. Adams, R. M. Bowman, J. M. Gregg, Nanotechnology 2007, 18, 035301.

25 V. Ya. Shur, A. Gruverman, V. P. Kuminov, N. A. Tonkachyova, Ferroelectrics 1990, $111,197$.

26 A. N. Kolmogorov, Izv. Akad. Nauk USSR, Ser. Math. 1937, 3, 355.

27 Y. Ishibashi, Y. Takagi, J. Phys. Soc. Jpn. 1971, 31, 506.

28 X. F. Du, I. W. Chen, Appl. Phys. Lett. 1998, 72, 1923.

29 A. K. Tagantsev, I. Stolichnov, N. Setter, J. S. Cross, M. Tsukada, Phys. Rev. B 2002, 66, 214109.

30 C. Kittel, Phys. Rev. 1951, 83, 458.

31 J. R. Taylor, Classical Mechanics, University Science Books, Sausalito, U.S.A. 2005.

32 M. Dawber, D. J. Jung, J. F. Scott, Appl. Phys. Lett. 2003, 82, 436.

33 Y. Kim, H. Han, W. Lee, S. Baik, D. Hesse, M. Alexe, Nano lett. 2010, 10, 1266.

34 G. Catalan, J. Seidel, R. Ramesh, J. F. Scott, Rev. Mod. Phys. 2012, 84, 119. 
35 G. T. Rado, R. W. Wright, W. H. Emerson, Phys. Rev. 1950, 80, 270.

36 L. Thomas, M. Hayashi, X. Jiang, R. Moriya, C. Rettner, S. Parkin, Science 2007, 315, 1553.

37 A. P. Malozemoff, J. C. DeLuca, Appl. Phys. Lett. 1975, 26, 719.

38 L. Thomas, R. Moriya, C. Rettner, S. S. P. Parkin, Science 2010, 330, 1810.

39 Y. Ehara, S. Yasui, J. Nagata, D. Kan, V. Anbusathaiah, T. Yamada, O. Sakata, H. Funakubo, V. Nagarajan, Appl. Phys. Lett. 2011, 99, 182906.

40 J. Li, B. Nagaraj, H. Liang, W. Cao, Chi. H. Lee, R. Ramesh, Appl. Phys. Lett. 2004, 84, 1174.

41 A. Q. Jiang, H. J. Lee, C. S. Hwang, J. F. Scott, Adv. Funct. Mater. 2012, 22, 192.

42 D. R. Callaby, J. Appl. Phys. 1965, 36, 2751.

43 R. C. Miller, A. Savage, J. Appl. Phys. 1961, 32, 714.

44 S. V. Kalinin, D. A. Bonnell, Phys. Rev. B 2001, 63, 125411. 